\title{
Intramuscular Stimulation (IMS)
}

\author{
Sang-Chul Lee and Young-Jae Kim \\ Additional information is available at the end of the chapter \\ http://dx.doi.org/10.5772/58565
}

\section{Introduction}

Chronic pain is common with relatively high incidence and low recovery rates [1]. Chronic pain can cause disability, mild to severe suffering and a serious problem to the health of the public. Chronic pain is localized to the musculoskeletal system in the majority of patients [2]. The most reported forms of chronic musculoskeletal pain are frequently back pain. However non-specific spinal disorders are not possible to identify a pathomorphological source of the problem despite a thorough diagnostic work-up such as simple radiography, computed tomography, magnetic resonance imaging, ultrasound, electromyography and nerve conduction test [1]. There are many potential causatives and aggravating factors associated with non-specific spinal disorders. Though laboratory and radiologic tests provide a myriad of information including the musculoskeletal and nerve system and give important clues for the diagnosis, structural abnormalities and clinical symptoms do not match commonly in clinical practice [3]. Unfortunately some patients do not improve despite administering conservative treatment and then the various interventional therapies, including medical treatment and/or surgery, and they find themselves in search of a more effective pain relief.

Deep dry needling is one of the alternative treatment modalities for these patients who do not respond to drug therapy and clinical intervention [4]. Previous clinical study demonstrated that dry needling into the trigger points (MTrPs) of myofascial pain syndrome is as effective as the injection of local anesthetics in inactivating them [5]. Especially, there appears to be growing interest in the intramuscular stimulation (IMS) for myofascial pain of radiculopathic origin developed by the Canadian physician Dr. Chan Gunn [6]. According to Gunn's approach, dry needling should be performed not only in muscle at the site of pain but also in the paraspinal muscles of the same spinal segment that innervates the painful muscles as causes and treatment targets of chronic pain. IMS is the technique for 
needle insertion and mechanical stimulation into trigger points or motor units of muscle belong to both anterior and posterior primary rami of spinal nerve root which require treatment.

In this chapter, we introduce IMS as an alternative and effective method for the management of chronic pain.

\section{Basic background for IMS}

Dry needling methods have empirically been developed to treat musculoskeletal disorders. In 1942, Dr. Janet Travell and colleagues firstly published the method by intramuscular infiltration with procaine hydrochloride [7]. The wider use of dry needling started after Lewit's publication [5], where it was emphasized that the needling effect was distinct from that of the injected substance and the effect of injections was primarily caused by the mechanical stimulation of myofascial trigger points (MTrPs) with the needle. In addition, in numerous randomized clinical trials [8-9], no difference was found between injections of different substances and dry needling in the treatment of MTrPs.

Several models of dry needling have developed during the last 3 decades. The radiculopathy model is based on empirical observations by Dr. Gunn[10], named IMS to distinguish this approach from other methods of dry needling. IMS technique is based on the premise that myofascial pain syndrome is always the result of peripheral neuropathy or radiculopathy, defined as a condition that causes disordered function in the peripheral nerve [6].

\section{Radiculopathic model of IMS}

In the radiculopathy model, based on Cannon and Rosenblueth's Law of Denervation Supersensitivity [11], denervated tissues develop supersensitivity. When a portion from a chain of nerve units is irritated, the receptor sensitivities to chemical stimuli in that point and the zones below it (muscles, skin, blood vessels, ligaments and tenoperiostea) become abnormally increased and these effects are maximized at the directly damaged sites [10]. The most common sites of supersensitivity are skeletal muscles. Indeed supersensitivity leads to muscle shortening when a nerve unit is injured, and by which myofascial pain syndrome is induced [10]. In the musculature, shortened muscles can physically cause a large variety of pain syndromes by its relentless pull on various structures [12] [Fig. 1] [Table 1]. Muscular evidence of radiculopathy is almost found in the distribution of both dorsal and ventral rami of affected segmental nerves. Shortening of the paraspinal muscles (particularly the multifidi muscles) innervated by dorsal ramus of affected segmental nerves leads to disk compression and narrowing of the intervertebral foramina, or direct pressure on the nerve root, which subsequently results in peripheral neuropathy and the development of supersensitive nociceptors and pain [Fig. 2]. 


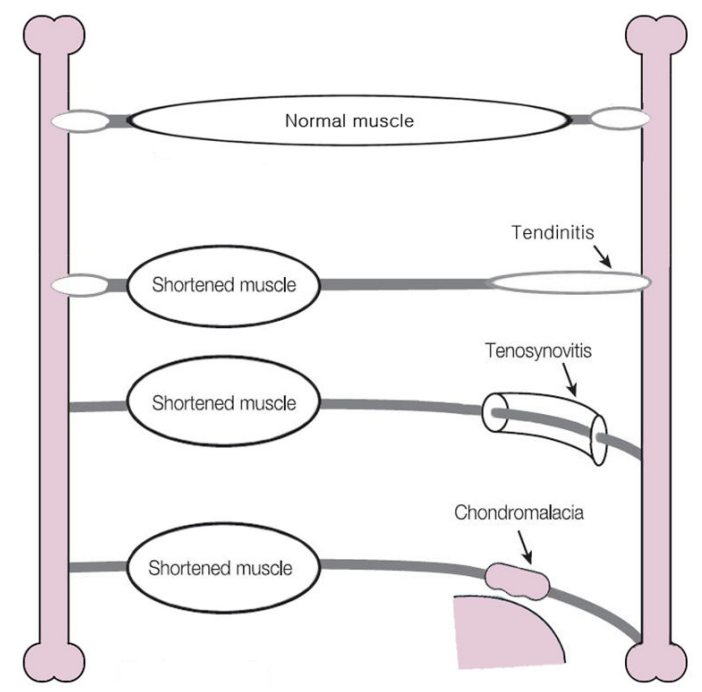

Figure 1. The shortened muscles cause the tendinitis, tenosynovitis and chondromalacia by increased traction at mechanically overloading the tendons and joints.

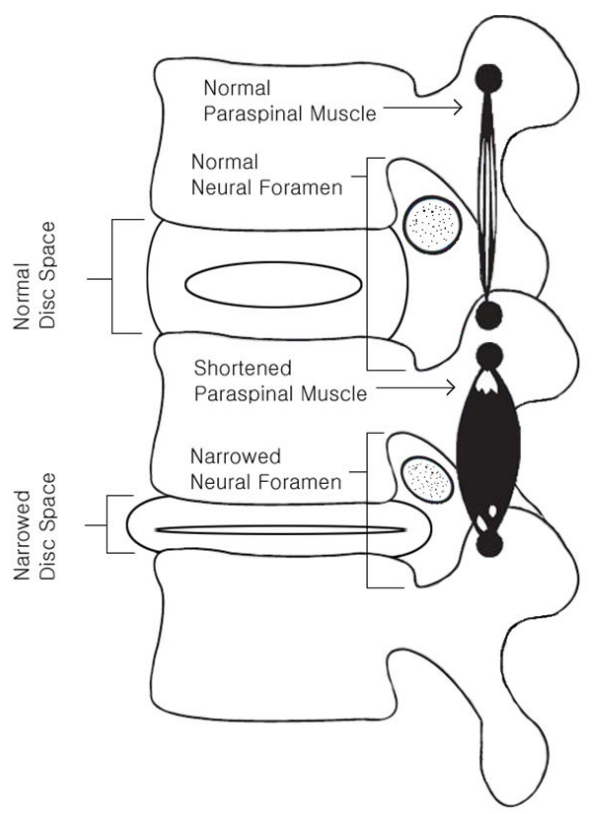

Figure 2. The shortened paraspinal muscles compress upon the nerve root by narrowed disc space and neural foramen. 
Radiculoapthy can be often accompanied by partial denervation. Chronic attrition from the spondylosis is the most common among the causes of nerve damage, such as trauma, metabolic, degenerative, toxic, and other conditions [13]. The spondylosis has the structural disintegration and morphologic alterations that occur in the intervertebral disc, with pathoanatomical changes in surrounding structures. The spinal nerve root, because of its vulnerable position, is notably prone to injury from pressure, stretch, angulation, and friction due to pathoanatomical changes in spondylosis. Other causes of radiculopathy, such as arachnoiditis, neuroma, and intraspinal tumors are much less common. The spondylosis increases with age, and causes repeated major and minor injuries to a segment nerve leading to unresolved clinical residuals which may, or may not, produce pain [14].

\begin{tabular}{|c|c|}
\hline Syndrome & Shortened muscle \\
\hline Achilles tendonitis & Gastrocnemii, soleus \\
\hline Bicipital tendonitis & Biceps brachii \\
\hline Bursitis, pre-patellar & Quadriceps femoris \\
\hline Capsulitis, frozen shoulder & All muscles acting on the shoulder \\
\hline Carpal tunnel syndrome & pronator teres, the sublimis bridge, Trophedema in the forearm and carpal tunnel \\
\hline Cervical fibrositis & Cervical paraspinal muscles \\
\hline Chondromalacia patellae & Quadriceps femoris \\
\hline De Quervain's tenosynovitis & Abductor pollicis longus, extensor pollicis brevis \\
\hline Facet syndrome & Muscles acting across the facet joint \\
\hline Fibromyalgia & Multisegmental (diffuse myofascial pain syndrome). \\
\hline Hallux valgus & Extensor hallucis longus and brevis \\
\hline Headaches- frontal & Upper trapezius, semispinalis capitis, occipitofrontalis \\
\hline Headaches-temporal & Temporalis, trapezius \\
\hline Headaches-vertex & Splenius capitis \& cervicis, upper trapezius, semispinalis capitis, occipitofrontalis \\
\hline Headaches-occipital & Sub-occipital muscles \\
\hline Infrapatellar tendonitis & Quadriceps femoris \\
\hline Intervertebral disc & Muscles acting across the disc space \\
\hline Juvenile kyphosis and scoliosis & Unbalanced paraspinal scoliosis muscles (e.g., iliocostalis thoracis and lumborum) \\
\hline Low back sprain & Paraspinal muscles \\
\hline Plantar fascitis & Flexor digitorum brevis, lumbricals \\
\hline Piriformis syndrome & Piriformis muscle \\
\hline Rotator cuff syndrome & Supra-and infraspinati, teres minor, subscapularis \\
\hline 'Shin splints' & Tibialis anterior \\
\hline Temporomandibular joint & Masseter, temporalis, pterygoids \\
\hline Tennis elbow & $\begin{array}{l}\text { Brachioradialis, carpi ulnaris, extensor carpi radialis brevis and longus, ext. } \\
\text { digitorum, anconeus, triceps. }\end{array}$ \\
\hline Torticollis (acute) & Splenius capitis \& cervicis. \\
\hline
\end{tabular}

Table 1. Common myofascial pain syndromes caused by the shortened muscle syndrome 
In addition, radiculopathy itself contributes to degenerative conditions. Neuropathy degrades the quality of collagen [15]. The amount of collagen in soft and skeletal tissues is also reduced. Because collagen lends strength to ligament, tendon, cartilage, and bone, neuropathy can expedite degeneration in weight-bearing and activity-stressed parts of the body which include the spine and joints.

Clinical features of radiculopathy differ from those of denervation such as loss of sensation and reflexes. The effects of radiculopathy vary according to the type of sensory, motor, autonomic, or mixed dysfunction and distribution of the nerve fibers involved.

\section{Clinical features of radiculopathy}

In radiculopathy, symptoms and signs are generally present in the territories of both posterior and anterior primary divisions of the affected nerve root. Clinical features of radiculopathy are projected to dermatomal, myotomal, and sclerotomal target structures supplied by the affected neural structure. The clinical characteristics can give rise to sensory, motor, autonomic, or mixed dysfunction.

Muscle shortening has painful spots on compression that are associated with hypersensitive palpable nodules in the taut band of skeletal muscle. The spots can cause tenderness, characteristic referred pain, motor dysfunction and autonomic phenomena. These can be tender, especially over motor points. Tender points can be found throughout the myotome and especially in paraspinal muscles.

Autonomic vasoconstriction of affected parts is colder in a noticeable manner. Increased permeability in blood vessels can lead to the trophedema that is edema in local subcutaneous tissue. The trophedema especially shows a characteristic feature like orange-peel skin over affected regions by rolling or squeezing an area of skin and subcutaneous tissue. The skin is tight and wrinkles absent. The consistency of subcutaneous tissue is firmer. The trophedema is not pitting to digital pressure, but to a blunt instrument pressure with the end of a matchstick. Excessive sweating as sudomotor activity may follow painful movements. The pilomotor reflex is often hyperactive and visible as goose-bumps in affected dermatomes.

The tendinous attachments to bone are thickened due to shortening muscle, which causes enthesopathy at the tenoperiosteal insertion.

\section{Diagnosis for radiculopathy}

The physical examination should always be preceded by a clinical history. It is important to inspect for any postural asymmetries, assess the range of motion for limitation, and examine the soft tissues for clinical features of radiculopathy. The spinal examination should be performed scrupulously according to segmental examination to elicit the signs that correspond to the affected spinal segments. The spinal segmental examination includes assessment for 
facet joint tenderness, tenderness to posteroanterior pressure on the spinous process, transverse pressure against the spinous process, and pressure against the interspinous ligamentum. This examination can identify the responsible spinal segments.

Because segmental radiculopathy primarily causes the significant changes in muscle, the examination of the segmental nerve supply to muscles is the clue to diagnosis. The changes in muscles are the most consistent increased muscle tone, tenderness over motor points and palpable taut bands, and result in restricting a range of joint motion. During examination according to the distribution of both dorsal and ventral rami of affected segmental nerves, each muscle must be palpated. Moreover, because many paraspinal muscles are compound and extend throughout most of the length of the vertebral column, the entire spine must be examined even when symptoms are localized to one region. The contracture caused by shortened muscles due to radiculopathy is invisible to X-rays, CT scans or MRI.

Laboratory and radiologic findings are generally not helpful for diagnosis of radiculopathy. Thermography reveals decreased skin temperature in affected dermatomes due to autonomic dysfunction. Other diagnostic observation is to find goose-bumps and orange-peel skin by rolling or squeezing an affected area. And the tenoperiosteal tenderness is present only when periosteal insertion is affected and is often painful to palpation only without giving the patient any pain spontaneously.

\section{Technique for IMS}

IMS is a system of dry needling that is based on a radiculopathy model for chronic pain. The key to IMS treatment is the release of muscle shortening. The fundamental Needling points for effective treatment are always situated to muscular motor points or musculotendinous junctions. These points generally coincide with palpable taut bands that are tender to digital pressure and are generally referred to as MTrPs. The muscles with tender points are generally shortened from contracture. Needling points generally belong to the same segmental level as presenting symptoms and signs. Tender points are distributed in a segmental or myotomal fashion, in muscles of both anterior and posterior primary rami which is indicative of radiculopathy. Practitioners purposely seek out tender and tight muscle bands in affected segments for needling.

The needles inserted in the plungers are made of a fine, flexible, solid and stainless steel like acupuncture needle [Fig 3,4]. Its lengths are 4, 6, and $8 \mathrm{~cm}$ (diameter: $0.25,0.3$ and $0.4 \mathrm{~mm}$ respectively). The absolute size of needle is dependent on the muscle and the depth of the motor point being treated. IMS needles are longer, finer, and whippier than hypodermic needles and are particularly suited for deep muscle exploration. The plunger is sterilized by autoclaving.

When the needling point is identified, standard precautionary techniques for asepsis are followed (hands scrubbed, no gloves, and skin cleansed with alcohol). The thumb and index 


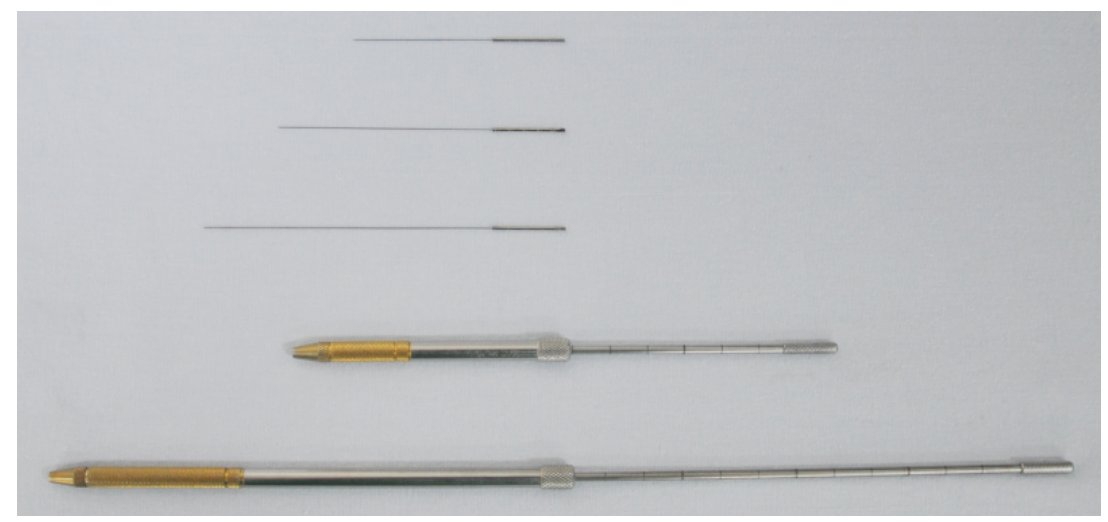

Figure 3. Types of needles and plungers according to the length of needles.

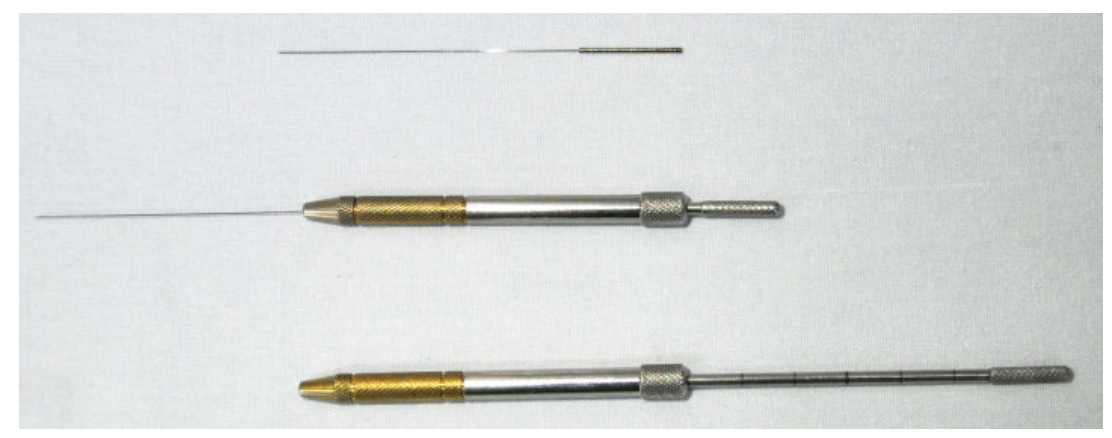

Figure 4. The needle is inserted in the plunger.

finger of the physician's nondominant hand holding plunger remain unmoved to guide the needle [Fig. 5]. The direction of needle insertions using plunger is perpendicular to the skin with the objective of penetrating the motor units. When the index finger of the physician's dominant hand on the non-needle end of plunger pushes the needle, it quickly penetrates the skin to $2 \sim 3 \mathrm{~mm}$ depth [Fig. $5 \mathrm{~A}$ ]. And then IMS needle is followed several times by pecking and twirling movements [Fig. 5 B]. Therefore IMS allows stimulation of deeper motor units by using a manual plunger for inserting, pecking and twirling of the needle.

The fine, flexible needle transmits feed-back information on the nature and consistency of the tissues that it is penetrating. When the needle penetrates normal muscle, it meets with little hindrance. When it penetrates a contracted muscle, there is firm resistance, and the needle is grasped by the muscle. When an attempt is made to withdraw the needle, the grasp resists withdrawal. Leaving the grasped needle in situ for 5 to 20 minutes can lead to the release of a persistent contracture. 

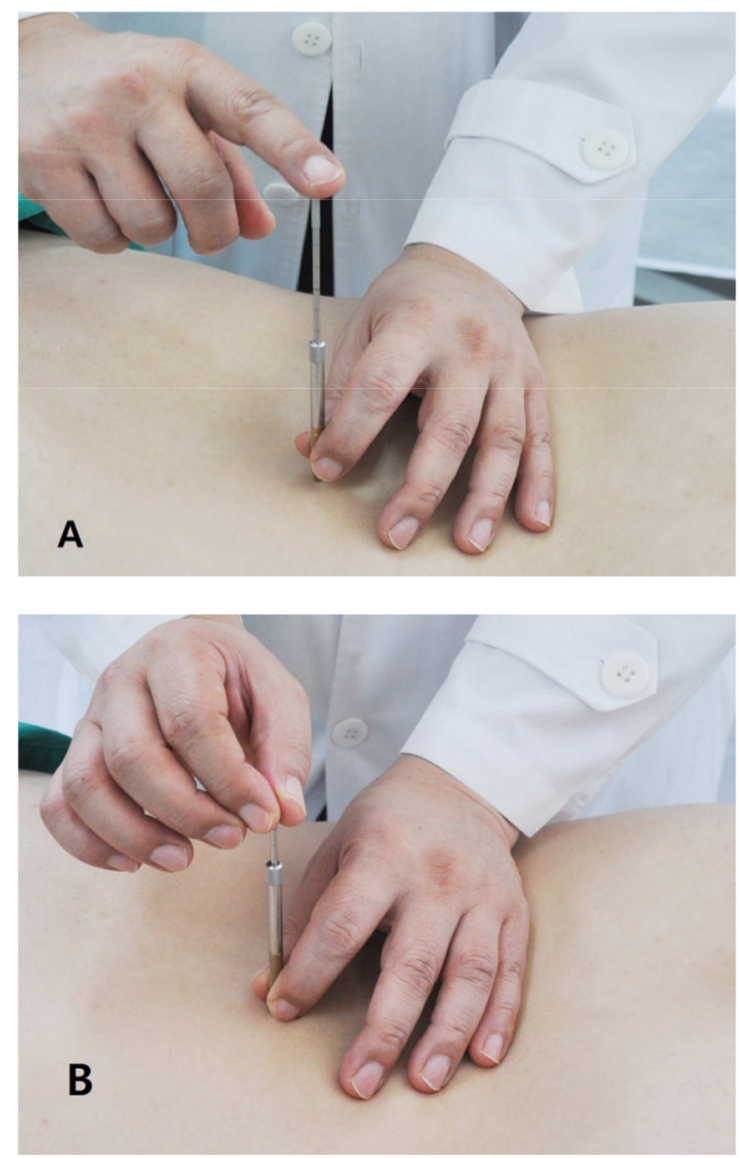

Figure 5. The technique of intramuscular stimulation. (A) Needle penetrates the skin. (B) Needle is pecked \& rotated.

Following needling, muscle contracture, vasoconstriction and tenderness can disappear within seconds or minutes. Other signs, like trophedema may diminish more gradually, sometimes even taking days to disappear, but ultimately, all signs vanish following successful treatment.

\section{The effect of IMS}

There is limited evidence supporting that IMS has an overall treatment effect when compared with standardised care. The result of the meta-analysis searching all studies regardless of the language to include all the available clinical evidence on IMS included 5 randomized controlled trials (RCT) [16, 17]. The represented conditions had MPS of upper and lower half body, MPS of the upper trapezius muscle, chronic shoulder pain, tension-type headaches and chronic 
lower back pain. However, the large scale, good quality placebo controlled researches in IMS are needed for RCTs because of the limited sample size and poor quality of these studies.

Release of these muscles by stimulation of motor units with dry needling is usually necessary to restore joint range and relieve pain [10].Dry needling can cause a decrease in spontaneous electrical amplitude and subsequent relaxation via either a direct local electrical stimulus or via a reflex mechanism [18]. As the needle is introduced into muscle tissue, it can cause a shortened muscle to visibly fasciculate and subsequently relax [10]. In addition as the muscle is injured by the needle then a "current of injury" follows, this current of injury was first described in 1797 by Galvani. Injury potentials of several microamperes are generated and can persist and provide stimulation for days until the miniature wounds heal. It was demonstrated that denervation supersensitivity in animal muscle may be reduced or abolished by electrical stimulation [19].

In addition, twirling the needle causes muscle fibers cling to the needle to wind around its shaft. The rotation of a needle grasped by muscle shortening can produce intense stimulation. Unlike traction or manipulation, this stimulation is very precise and intense because the needle is precisely placed in a taut muscle band. Rotational motion is converted to linear motion which shortens the muscle fibers locally. This shortening of muscle fibers by twirling the needle activates muscle spindles and Golgi Tendon Organs and may cause subsequent muscle relaxation via local spinal reflexes. Therefore the needle rotation may induce neuroplastic changes as the pulling of collagen fibers and the transduction of the mechanical signal into fibroblasts can lead to a wide variety of cellular and extracellular events, including mechanoreceptor and nociceptor activation and eventually to neuropeptide liberation [20].

Needling also induces a sympatholytic effect that spreads throughout the body segment, releasing vasoconstriction. Pain in muscles, tendons and joints caused by excessive muscle tension is eased when the shortened muscles are relaxed. Subjective improvement can objectively be confirmed the increase in the motion of range, reduction of joint effusion and any decrease in muscle tenderness within minutes.

Dry needling delivers to the injured area the platelet-derived growth factor (PDGF) which induces deoxyribonucleic acid (DNA) synthesis and stimulates collagen formation [21].

One intervention of dry needle stimulation to a single MTrP evokes short term segmental antinociceptive effects [22]. MTrP stimulation by dry needling may evoke antinociceptive effects by modulating segmental mechanisms.

\section{Adverse effect of IMS}

No serious adverse effects were reported and the frequency of minor adverse effects occurred $[23,24,25]$. Several adverse effects associated specifically with dry needling include soreness after needling, local hemorrhages at the needling site and syncopal responses, and rarely reported a pneumothorax. Post-needling soreness is the most common due to local hemorrhages at the needling site and can be prevented by sufficient compression after treatment [26]. 
In addition, the thinner pointed-tipped IMS needles used for inserting trigger points can induce less tissue injuries than the thick and hollow needles with a beveled and cutting edge.

\section{Difference between IMS and Acupuncture}

IMS differs from chinese traditional acupuncture using superficial dry needling [table 2]. Unlike chinese traditional acupuncture, IMS requires a medical examination searching for early signs of radiculopathy, the knowledge of anatomy and a medical diagnosis, and uses neuroanatomical points that are found in a segmental pattern, instead of using traditional acupuncture points that is non-scientific meridians.

\begin{tabular}{lll}
\hline & Intramuscular stimulation & Acupuncture \\
\hline Theory & $\begin{array}{l}\text { Western medicine's understanding of the } \\
\text { neurophysiology of pain and a greater } \\
\text { knowledge of anatomy, muscle balancing }\end{array}$ & $\begin{array}{l}\text { An ancient Chinese philosophy into non- } \\
\text { scientific meridians }\end{array}$ \\
& and biomechanics & \\
\hline Diagnosis & Medical examination, and laboratory and X- Inspection and pulse diagnosis \\
& ray test & \\
\hline needle placement & Into deep muscles & Into the superficial or subcutaneous tissues \\
\hline Needling techniques & $\begin{array}{l}\text { Pecking and twirling movements with } \\
\text { plunger }\end{array}$ & Straight insertion \\
\hline Treatment site & $\begin{array}{l}\text { Motor points of the shortened muscle, and } \\
\text { its corresponding spinal segment }\end{array}$ & pre-mapped out points in the body and \\
& Subjective and objective effects are usually & Any effects are not experienced. \\
\hline Effect of treatment & experienced right after the treatment & \\
\hline
\end{tabular}

Table 2. The difference between IMS and Acupuncture

\section{Conclusion of IMS}

Many patients suffering from chronic pain have associated musculoskeletal pain which is not readily detectable with laboratory and radiologic tests. This pain may be due to both peripheral and central sensitization mechanisms. A radiculopathy model is offered to explain these syndromes. This model enables many apparently dissimilar musculoskeletal pain syndromes to be grouped under one etiologic classification such as radiculopathy. IMS is an alternative system of dry needling for treatment of chronic pain based on neurophysiologic concepts. 


\section{Author details}

Sang-Chul Lee ${ }^{1}$ and Young-Jae Kim²

1 Department of Anesthesiology and Pain Medicine, Seoul National University, Seoul, South Korea

2 Department of Anesthesiology and Pain Medicine, Busan Paik Hospital, College of Medicine, Inje University, Busan, South Korea

\section{References}

[1] Balagué F, Mannion AF, Pellisé F, Cedraschi C. Non-specific low back pain. Lancet 2012; 379: 482-91.

[2] Manchikanti L, Boswell MV, Singh V, Derby R, Fellows B, Falco FJ, et al. Comprehensive review of neurophysiologic basis and diagnostic interventions in managing chronic spinal pain. Pain Physician. 2009 ;12: E71-120.

[3] Elliott AM, Smith BH, Hannaford PC, Smith WC, Chambers WA. The course of chronic pain in the community: results of a 4-year follow-up study. Pain. 2002;99:299-307.

[4] Gunn CC. Dry needling of muscle motor points for chronic low back pain. Spine 1980; 5: 279-91.

[5] Lewit K. The needle effect in the relief of myofascial pain. Pain 1979;6:83-90.

[6] Gunn CC. Radiculopathic pain: diagnosis and treatment of segmental irritation or sensitization. J Musculoskelet Pain 1997;5:119-34.

[7] Travell J, Rinzler S, Herman M. Pain and disability of the shoulder and arm: treatment by intramuscular infiltration with procaine hydrochloride. JAMA 1942;120:41722.

[8] Hong CZ. Lidocaine injection versus dry needling to myofascial trigger point. The importance of the local twitch response. Am J Phys Med Rehabil 1994;73:256-63.

[9] Cummings TM, White AR. Needling therapies in the management of myofascial trigger point pain: a systematic review. Arch Phys Med Rehabil 2001;82:986-92.

[10] Gunn CC. The Gunn approach to the treatment of chronic pain. 2nd Ed. New York: Churchill Livingstone; 1996:1-19

[11] Cannon WB, Rosenblueth A. The supersensitivity of denervated structure, a law of denervation. New York, MacMillan. 1949, pp 136-71 
[12] Gunn CC: The mechanical manifestations of neuropathic pain. Annals of Sports Medicine 1990;5:138-141.

[13] Gunn CC. Prespondylosis and some pain syndrome following denervation supersensitivity. Spine 1978; 5: 185-92.

[14] Sola, AE: Treatment of myofasical pain syndromes. Advances in Pain Research and Therapy. Edited by C Benedetti, CR Chapman, and G Morrica. Raven Press, New York, 1984, Vol. 7, pp. 467-85.

[15] Klein L, Dawson MH, Heiple KG: Turnover of collagen in the adult rat after denervation. J Bone Jt Surgery ;59A:1065-7.

[16] Lim SM, Seo KH, Cho B, Ahn K, Park YH. A systematic review of the effectiveness and safety of intramuscular stimulation therapy. J Korean Med Assoc 2011;54:1070 80 .

[17] Couto C, de Souza IC, Torres IL, Fregni F, Caumo W. Paraspinal stimulation combined with trigger point needling and needle rotation for the treatment of myofascial pain: a randomized sham-controlled clinical trial. Clin J Pain 2014;30:214-23.

[18] Chen J T, Chung K C, Hou C R, Kuan C R, Chen C R, Hong C Z. Inhibitory effect of dry needling on spontaneous electrical activity recorded from myofascial trigger points of rabbit skeletal muscle. Am J Phys Med Rehabil 2001;80:729-35.

[19] Lomo T, Massoulie J, Vigny M: Stimulation of denervated rat soleus muscle with fast and slow activity patterns induces different expression of acetylcholinesterase molecular forms. J Neurosci 1985;5:1180-7.

[20] Audette JF, Wang F, Smith H. Bilateral activation of motor unit potentials with unilateral needle stimulation of active myofascial trigger points. Am J Phys Med Rehabil 2004;83:368-74.

[21] Ross R, Vogel A. The platelet-derived growth factor. Cell 1978;4.203-10.

[22] Srbely JZ, Dickey JP, Lee D, Lowerison M. Dry needle stimulation of myofascial trigger points evokes segmental anti-nociceptive effects. J Rehabil Med 2010;42:463-8.

[23] $\mathrm{Ga} \mathrm{H}$, Choi JH, Park $\mathrm{CH}$, Yoon HJ. Dry needling of trigger points with and without paraspinal needling in myofascial pain syndromes in elderly patients. J Altern Complement Med 2007;13:617-24.

[24] Huguenin L, Brukner PD, McCrory P, Smith P, Wajswelner H, Bennell K. Effect of dry needling of gluteal muscles on straight leg raise: a randomised, placebo controlled, double blind trial. Br J Sports Med 2005;39:84 -90.

[25] Simons DG, Travell JG, Simons LS. Travell \& Simons' Myofascial Pain and Dysfunction: The Trigger Point Manual, vol 1: Upper Half of Body, 2nd ed., Baltimore: Williams \& Wilkins, 1999:5. 
[26] Kalichman L, Vulfsons S. Dry needling in the management of musculoskeletal pain J Am Board Fam Med 2010;23:640-6. 
